\title{
Digitalisierung der Branchen in Deutschland - eine empirische Erhebung
}

\author{
Der hier vorgestellte Digitalisierungsindex misst den Grad der Digitalisierung von Unternehmen in \\ Deutschland, differenziert dabei nach Branchen und ordnet den Digitalisierungsgrad in Kategorien \\ ein. Es zeigt sich, dass die Informations- und Kommunikationstechnologie mit Abstand am \\ stärksten digitalisiert ist. Auch der forschungsstarke Fahrzeugbau ist stark überdurchschnittlich \\ digitalisiert. Schlusslichter sind der Tourismus und das Sonstige Produzierende Gewerbe.
}

Die Messung der Digitalisierung der Wirtschaft, also des Standes und der Entwicklung der digitalen Transformation, ist nicht trivial. Daher ist es auch nicht unkompliziert möglich, einen möglichen „Digitalisierungsschub“ zu quantifizieren. Schon vor der Pandemie gab es für Deutschland zahlreiche Publikationen, die sich mit dem Status quo der Digitalisierung von Unternehmen, des Staates oder der Gesellschaft beschäftigt haben. Exemplarisch zu nennen ist hier die jährliche Erhebung der Europäischen Kommission (2020) im Digital Economy and Society Index DESI. Der DESI vergleicht die europäischen Mitgliedstaaten in wesentlichen Kernindikatoren der Digitalisierung von Wirtschaft, Staat und Gesellschaft. Der hier gewährte Blick auf die Unternehmen ist jedoch einseitig: Es wird kaum erfasst, wie Digitalisierung in Unternehmen eigentlich abläuft, wie digital Prozesse, Produkte und Geschäftsmodelle bereits sind. Solche Aspekte können Befragungen abdecken (z. B. Azkan et al., 2019), die jedoch oft einmalig durchgeführt werden und damit keine Entwicklung im Zeitablauf darstellen. Solche Befragungen bilden oft nur die interne Digitalisierung von Unternehmen ab und bieten keinen Blick auf die Rahmenbedingungen, innerhalb derer die Unternehmen zwangsläufig operieren.

Vor diesem Hintergrund beschäftigt sich das Projekt „Entwicklung und Messung der Digitalisierung der Wirtschaft am Standort Deutschland“, das durch das Bundesministerium für Wirtschaft und Energie gefördert wird, mit der Schaffung einer jährlich wiederholbaren Empirie zur Digitalisierung der Unternehmen in Deutschland. Dabei berücksichtigt dieser Digitalisierungsindex explizit die unternehmensinterne Perspektive wie auch das unterneh-

(C) Der/die Autor:in(nen) 2021. Open Access: Dieser Artikel wird unter der Creative Commons Namensnennung 4.0 International Lizenz veröffentlicht (creativecommons.org/licenses/by/4.0/deed.de).

Open Access wird durch die ZBW - Leibniz-Informationszentrum Wirtschaft gefördert. merische Umfeld für die Digitalisierung. Dieser inhaltliche Schwerpunkt wie auch die intertemporale Vergleichbarkeit der Ergebnisse des Digitalisierungsindex legen den Grundstein für politische Ableitungen zur Förderung der Digitalisierung der Wirtschaft in Deutschland.

\section{Zur Methodik des Digitalisierungsindex}

Ziel des Digitalisierungsindex ist es, eine jährlich wiederholbare Empirie zu schaffen, welche den Stand der Digitalisierung der Wirtschaft in Deutschland abbildet. Zu diesem Zweck werden einzelne Indikatoren verwendet, die jeweils unterschiedliche Aspekte der Digitalisierung erfassen und dann zu einem Indexwert zusammengeführt werden. In dem Projektkonsortium sind das Leibniz Zentrum für Europäische Wirtschaftsforschung (ZEW; Konsortialführer), das Institut der deutschen Wirtschaft (IW), die IW Consult, das Forschungsinstitut für Rationalisierung (FIR) an der RWTH Aachen und das Deutsche Institut für Wirtschaftsforschung (DIW) involviert. Der Digitalisierungsindex lässt sich neben der Erhebung auf gesamtdeutscher Ebene nach Unternehmensgrößenklassen, Bundeslandgruppen, Regionstypen sowie Branchen ausweisen. Ins-

Dr. Vera Demary leitet das Kompetenzfeld Strukturwandel und Wettbewerb im Institut der deutschen Wirtschaft in Köln.

Dr. Henry Goecke leitet die Forschungsgruppe Big Data Analytics im Institut der deutschen Wirtschaft in Köln. 
gesamt besteht der Index aus vier Ebenen: ${ }^{1}$ Der Digitalisierungsindex auf der obersten Ebene lässt sich auf der Ebene darunter in die Subindizes "unternehmensintern“ und „unternehmensextern" unterteilen. Diese adressieren, dass die Digitalisierung der Wirtschaft zwar primär innerhalb der Unternehmen erfolgt, dabei aber intensiv von den Rahmenbedingungen für Digitalisierung beeinflusst wird, die von außen vorgegeben sind. Auf einer weiteren Ebene werden die beiden Subindizes in jeweils fünf thematische Kategorien gesplittet, die verschiedene inhaltliche Aspekte der Digitalisierung gruppieren. Beim Subindex "unternehmensintern" sind dies die Kategorien Prozesse, Produkte, Geschäftsmodelle, Qualifizierung sowie Forschungs- und Innovationsaktivitäten. Zum Subindex „unternehmensextern“ zählen die Kategorien technische Infrastruktur, administrativ-rechtliche Rahmenbedingungen, Gesellschaft, Humankapital und Innovationslandschaft. Schließlich werden auf der untersten Ebene diesen Kategorien Indikatoren zugeordnet. Insgesamt werden zur Messung des Standes der Digitalisierung der Wirtschaft in Deutschland 37 Indikatoren berücksichtigt. Bei der Auswahl der Indikatoren wurde darauf geachtet, dass die einzelnen Indikatoren inhaltlich möglichst gut die jeweiligen Kategorien und damit auch die beiden Subindizes abbilden und untereinander möglichst überschneidungsfrei sind.

Die einzelnen ausgewählten Indikatoren liegen in verschiedenen Einheiten und mit unterschiedlichen Skalen vor (z.B. in \% oder als Anteil an einer anderen Größe). Um beispielsweise die Werte für unterschiedlich große Branchengruppen sinnvoll miteinander vergleichen zu können, werden die Indikatorwerte zunächst, wo möglich und notwendig, auf eine andere sinnvolle Größe bezogen (z. B. die absolute Zahl an Patenten auf die sozialversicherungspflichtig Beschäftigten). Um auch eine Vergleichbarkeit zwischen den Indikatoren über die Zeit und innerhalb der verschiedenen Differenzierungsebenen (wie etwa Branchen oder Bundeslandgruppen) zu ermöglichen, wird im ersten Jahr der Betrachtung (Erhebungsjahr 2020) die Ausprägung für Deutschland insgesamt für jeden Indikator auf 100 normiert. Diese Vorgehensweise führt dazu, dass auf der Ebene von Deutschland im ersten Betrachtungsjahr alle Indikatoren und damit auch der Gesamtindex den Wert 100 erhalten. Damit ist im Erhebungsjahr 2020 keine Aussage über den Status quo der deutschen Wirtschaft insgesamt möglich. Ab dem zweiten Jahr der Betrachtung (ab Erhebungsjahr 2021) wird jeder Indikatorwert für Deutschland dann ausgehend vom Wert des ersten Jahres berechnet $(2020=100)$. Er zeigt dann an, ob die einzelnen Indikatoren für Deutschland eine posi-

1 Eine ausführliche Erläuterung der Methodik findet sich in Büchel et al. (2020). tive (Wert $>100$ ) oder negative Entwicklung der Digitalisierung darstellt (Wert $<100)$. Für jeden Indikator werden die Ausprägungen in den vier Differenzierungsebenen in Relation zum jeweiligen Durchschnitt gesetzt. Um die Indikatoren zu einem Indexwert zu aggregieren, sind an zwei Stellen Gewichtungen notwendig. Innerhalb der zehn oben genannten Kategorien erfolgt grundsätzlich eine Gleichgewichtung der einzelnen jeweils enthaltenen Indikatoren. Bei empirisch vorliegenden Korrelationen sowie einer nicht vermeidbaren hohen inhaltlichen Überschneidung zwischen einzelnen Indikatoren einer Kategorie werden diese jedoch untergewichtet, um den in ihnen enthaltenen Effekt nicht mehrfach aufzunehmen. Die Gewichtung der zehn Kategorien, die notwendig ist, um die Werte für die beiden Subindizes und den Gesamtindex zu erhalten, wurde mithilfe einer Unternehmensbefragung unter rund 2.000 Unternehmen im Rahmen des IW-Zukunftspanels ermittelt. In dieser Befragung wurden die Unternehmen zur Wichtigkeit der einzelnen Kategorien für die Digitalisierung ihres Unternehmens befragt.

Die einzelnen Indikatoren liegen nicht immer auf allen Differenzierungsebenen vor - beispielsweise gibt es sinnvollerweise keine Differenzierung der Breitbandverfügbarkeit nach unterschiedlich großen Unternehmen. Die fehlenden Indikatoren und ihre Gewichte werden bei der Berechnung der spezifischen Indexwerte für die Differenzierungsebenen (Branchen, Unternehmensgrößenklassen, Bundeslandgruppen und Regionstypen) nicht berücksichtigt. In der Folge ist der Erklärungsgehalt des Index auf Deutschlandebene höher als auf den vier Differenzierungsebenen, da er der einzige Indexwert ist, in den alle 37 Indikatoren eingehen. Ein Vergleich der Werte für Deutschland beispielsweise mit den Werten für eine Branche ist aufgrund der unterschiedlichen Indikatoren nicht möglich. Stattdessen wird für die vier Differenzierungsebenen jeweils ein Durchschnittswert gebildet, der als Vergleichsgröße verwendet werden kann (z. B. der Durchschnittswert der zehn Branchengruppen). Dieser Wert wird im ersten Betrachtungsjahr ebenfalls auf 100 normiert, und um diesen Wert bewegen sich die Indexwerte der einzelnen Differenzierungsgruppen.

\section{Digitalisierung in den Branchen sehr unterschiedlich}

Die Gesamtergebnisse des Digitalisierungsindex auf Branchenebene zeigen, dass zwischen den zehn betrachteten Branchengruppen eine große Heterogenität vorliegt (vgl. Abbildung 1). Bei einem durchschnittlichen gewichteten Punktwert aller betrachteten Branchen von 100 beträgt die maximale Abweichung nach oben 173 Punkte, nach unten sind es rund 44 Punkte. Das beste Ergebnis erzielt - wenig überraschend - die Branche In- 
formations- und Kommunikationstechnologie (IKT) vor dem Fahrzeugbau sowie der Branchengruppe Elektrotechnik und Maschinenbau. Die geringsten Punktzahlen im Digitalisierungsindex erzielten das Sonstige Produzierende Gewerbe (z. B. Baugewerbe), die Tourismusbranche und das Sonstige Verarbeitende Gewerbe (z. B. die Herstellung von Textilien).

Um genauer ergründen zu können, wie dieses Bild der Digitalisierung der verschiedenen Branchengruppen zustande kommt, werden sie im Folgenden etwas detaillierter betrachtet. Schon beim Blick auf die Ergebnisse in den sechs für Branchengruppen relevanten Kategorien wird deutlich, dass so gut wie alle Branchen eindeutige Stärken und zumindest relative Schwächen aufweisen (vgl. Abbildung 2). Diese bilden klare Ansatzpunkte für Verbesserungen in der Digitalisierung der Unternehmen.

\section{Vorreiter IKT}

Die IKT-Branche bildet das Rückgrat der Digitalisierung der deutschen Wirtschaft. Die Branche entwickelt neue digitale Technologien und unterstützt andere Unternehmen bei deren Implementierung und Anwendung. Damit verbunden ist eine Vorreiterrolle der Branche in der Digitalisierung, die sich in dem weit überdurchschnittlichen Abschneiden in allen Kategorien des Digitalisierungsindex äußert. Besonders stark sticht die unternehmensexterne Kategorie Innovationslandschaft heraus, worunter die Indikatoren FuE-/Innovationskooperationen sowie digitale Start-ups zusammengefasst werden. Die FuE-/ Innovationskooperationen erfassen die Unternehmen, die innerhalb eines Dreijahreszeitraums im Rahmen von FuE- oder anderen Innovationsvorhaben an gemeinsamen Aktivitäten mit anderen Unternehmen teilgenommen haben. IKT landet hier mit knapp $26 \%$ deutlich über dem Durchschnitt der Branchen von rund $12 \%$ und an zweiter Stelle nach den Unternehmen der Branchengruppe Elektrotechnik und Maschinenbau. Noch deutlicher getrieben wird das sehr gute Abschneiden der IKT-Branche in dieser Kategorie allerdings durch den Indikator digitale Start-ups, der die Gründungen mit digitalen Geschäftsmodellen abbildet. Dies liegt in der Natur der Sache, weil Unternehmen mit digitalen Dienstleistungen, wie Programmierung, Datenanalyse und ähnliches, der IKT-Branche zuzuordnen sind und viele digitale Geschäftsmodelle solche digitalen Dienstleistungen beinhalten. Die IKTBranche erzielt hier einen Wert von etwa 8,3 Gründungen pro 10.000 sozialversicherungspflichtig Beschäftigten. Der Durchschnittswert der Branchen liegt hingegen bei gerade einmal 0,9.

Darüber hinaus weist die IKT-Branche in der Kategorie Qualifizierung weit überdurchschnittliche Werte auf. Die-

\section{Abbildung 1}

Ergebnisse des Digitalisierungsindex 2020 nach

\section{Branchen}

in Indexpunkten, gewichteter Durchschnitt der Branchen = 100

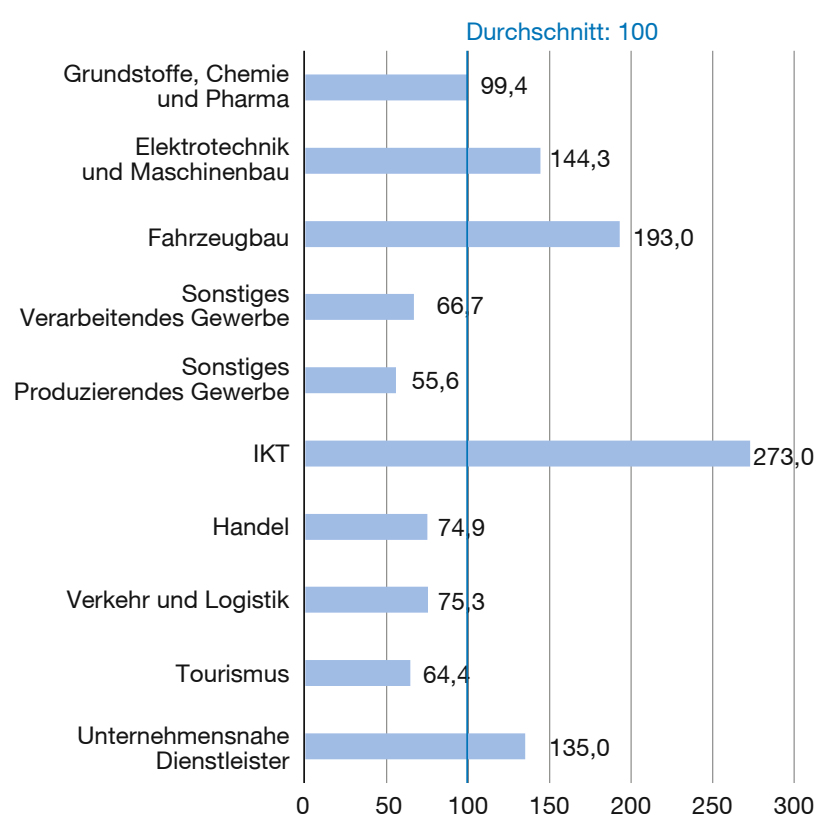

Quelle: Institut der deutschen Wirtschaft, IW Consult.

ses Ergebnis wird durch Werte der drei hier enthaltenen Indikatoren Weiterbildung IT-Fachkräfte, Weiterbildung IT-Anwendende und Beschäftigung in Digitalisierungsberufen getragen, die allesamt deutlich überdurchschnittlich sind. Mit 45,9\% ist der Anteil der sozialversicherungspflichtig Beschäftigten in Digitalisierungsberufen in der IKT-Branche besonders hoch - der Durchschnitt der Branchen liegt bei 11,9\%. Weiterbildungen für IT-Fachkräfte bieten rund $61,0 \%$ der Unternehmen in der IKT-Branche an. Demgegenüber sind es lediglich 13,7\% im Durchschnitt der Branchen. Die IKT-Branche zeigt hier sehr eindeutig, dass sie als Unterstützerin der Digitalisierung anderer Branchen Fachpersonal benötigt, das spezifische digitale Kompetenzen aufweist, die durch Weiterbildung gefestigt und ausgebaut werden. Diese Vorreiter- und Multiplikatorenrolle bestätigen auch die Ergebnisse der IKT-Branche in der Kategorie Produkte und den zugehörigen Indikatoren: 53,1 \% der Umsätze entfallen beispielsweise auf rein digitale Produkte. Im Durchschnitt der Branchen sind es lediglich 12,7\%.

\section{Mit Forschung und Innovation zu gutem Ergebnis}

Auf den Rängen zwei und drei hinsichtlich der Digitalisierung liegen der Fahrzeugbau und die Branchen- 


\section{Abbildung 2}

Ergebnisse des Digitalisierungsindex 2020 nach Kategorien und Branchen

in Indexpunkten, gewichteter Durchschnitt der Branchen = 100

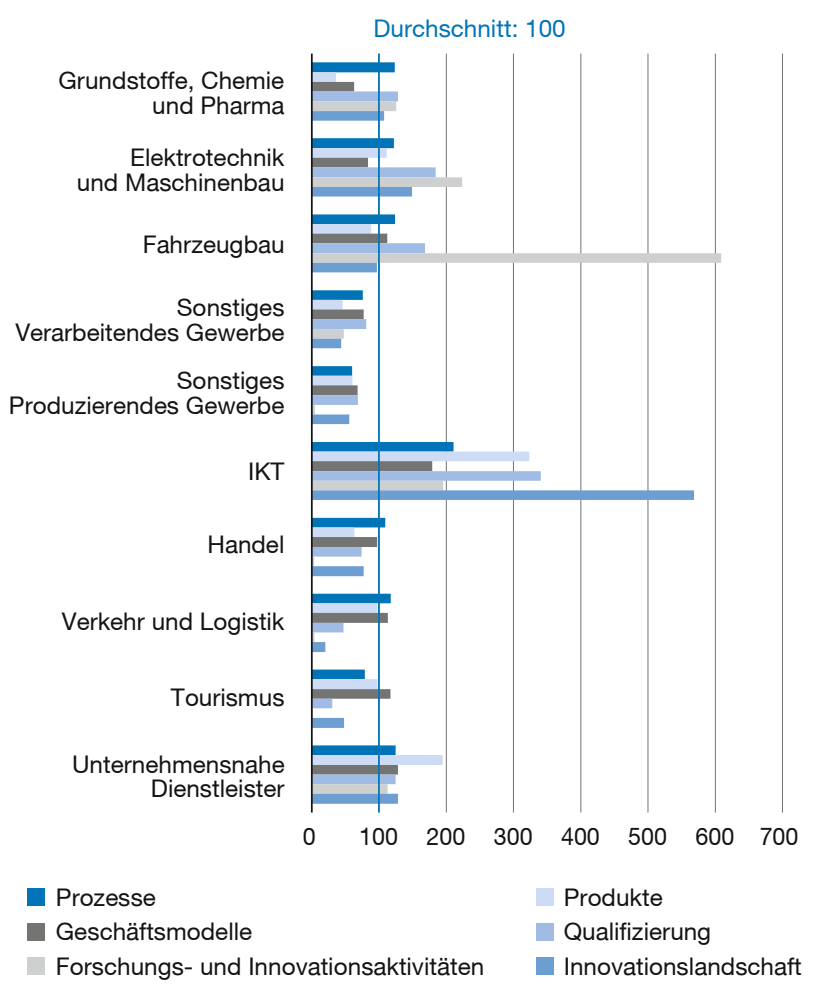

Quelle: Institut der deutschen Wirtschaft, IW Consult.

gruppe Elektrotechnik und Maschinenbau. Besonders auffallend in beiden Branchengruppen ist das überdurchschnittliche Abschneiden in der Kategorie Forschungs- und Innovationsaktivitäten: Mit Werten von rund 609 (Fahrzeugbau) beziehungsweise knapp 224 (Elektrotechnik und Maschinenbau) verdeutlichen diese Branchengruppen bei einem Durchschnitt der Branchen von 100, besonders forschungs- und innovationsaffin zu sein. Dieses Ergebnis wird vor allem im Fahrzeugbau durch das überragende Abschneiden beim Indikator digitalisierungsaffine Patente getragen. Solche Patente setzen in Europa eine gewisse Technizität voraus - Software ist hier nicht patentierbar. Pro 10.000 sozialversicherungspflichtig Beschäftigten weist der Fahrzeugbau hier knapp 41,6 solcher Patente auf. Zum Vergleich: Im Durchschnitt aller betrachteten Branchen sind es etwa 3,8 digitalisierungsaffine Patente pro 10.000 Beschäftigte. Der Fahrzeugbau liegt in diesem Indikator mit weitem Abstand vor allen anderen Branchen - die Branchengruppe Elektrotechnik und Maschinenbau schneidet ähnlich wie IKT ab und belegt mit einem Wert von 8,4 den dritten Rang.
Aber nicht nur der formelle Innovationsoutput macht die Stärke des Fahrzeugbaus und der Elektrotechnik und des Maschinenbaus in Forschungs- und Innovationsaktivitäten aus: Dazu kommt eine überdurchschnittliche Bedeutung des Forschungs- und Entwicklungspersonals in den Unternehmen. Im Fahrzeugbau sind rund 1.283 von 10.000 sozialversicherungspflichtig Beschäftigten in der FuE tätig. In der Elektrotechnik und dem Maschinenbau beträgt dieser Wert 671. Der Durchschnitt aller Branchen macht gerade mal 223 Personen in FuE pro 10.000 Beschäftigten aus.

Zu diesem sehr guten Abschneiden der beiden Branchengruppen in der Kategorie Forschungs- und Innovationsaktivitäten kommen überdurchschnittliche Ergebnisse in der Kategorie Qualifizierung. So bieten beispielsweise 29,9\% der Unternehmen des Fahrzeugbaus und 24,8\% der Unternehmen aus Elektrotechnik und Maschinenbau Weiterbildungen für IT-Fachkräfte an - bei einem Branchenschnitt von $13,7 \%$. Für IT-Anwendende sind die Werte, bei einem Branchendurchschnitt von gut $30 \%$ der Unternehmen, ebenfalls überdurchschnittlich - jedoch in einer umgekehrten Verteilung (die Branchengruppe Elektrotechnik und Maschinenbau liegt bei knapp 45\% wohingegen der Fahrzeugbau auf etwa $41 \%$ kommt).

\section{Ein gutes Mittelfeld}

Die Branchengruppe der unternehmensnahen Dienstleistungen - dazu gehören z.B. Steuerberatungen, Marktforschungsunternehmen und Ingenieurbüros - liegt mit einem Indexwert von 135 auf Rang 4 im Digitalisierungsindex. Auffallend ist bei dieser Branchengruppe vor allem die große Homogenität der Ergebnisse in den verschiedenen Kategorien (vgl. Abbildung 2). Während andere Branchengruppen deutliche Stärken und Schwächen aufzeigen, sind die Abstände der Kategorien bei den unternehmensnahen Dienstleistern gering und die Resultate überdurchschnittlich. Lediglich die Kategorie Produkte sticht besonders hervor. Besonders auffallend ist der Indikator Produkte mit digitalen Komponenten in dieser Kategorie: Die unternehmensnahen Dienstleister erreichen hier einen Anteil dieser Produkte am Umsatz von 16,7\%, dem zweithöchsten Anteil aller Branchen (Durchschnitt: 7,4 \%). Ebenfalls im Mittelfeld findet sich mit einem Indexwert von 99,4 die Branchengruppe Grundstoffe, Chemie und Phar$\mathrm{ma}$, in der die Kategorien Produkte und Geschäftsmodelle hinter den durchschnittlichen Ergebnissen der anderen Kategorien zurückbleiben. Angesichts der Erzeugnisse der Branche sind diese Resultate nachvollziehbar.

\section{Wo noch Nachholbedarf besteht}

Die übrigen fünf Branchengruppen weisen beim Digitalisierungsindex Ergebnisse wesentlich unterhalb des 
Durchschnitts der Branchen auf (vgl. Abbildung 1). Insbesondere in den Branchengruppen sonstiges Produzierendes Gewerbe - hierzu zählen z. B. Energie- und Wasserversorgung - sowie Sonstiges Verarbeitendes Gewerbe (beispielsweise Textil- und Papierherstellung) liegen die Ergebnisse in allen Kategorien unterhalb des Durchschnitts der Branchen. Einen besonders niedrigen Wert, wenn auch nicht den niedrigsten, erreicht das sonstige Produzierende Gewerbe in der Kategorie Forschungsund Innovationsaktivitäten. Die Branchengruppe ist bei allen drei hier enthaltenen Indikatoren FuE-Ausgaben Unternehmen, FuE-Personal Unternehmen und digitalisierungsaffine Patente Unternehmen stark unterdurchschnittlich. Beide genannten Branchengruppen erzielen auch in der Kategorie Innovationslandschaft geringe Werte. Dies wird in beiden Fällen insbesondere durch den Indikator digitale Start-ups verursacht. Sowohl im Sonstigen Produzierenden Gewerbe als auch im Sonstigen Verarbeitenden Gewerbe gibt es nur etwa 0,1 solcher digitalen Start-ups pro 10.000 Beschäftigten. Im Durchschnitt der Branchen liegt der Wert bei 0,9. Im Unterschied zu diesen beiden Branchengruppen zeigt die Branche Tourismus, die ebenfalls zu den drei Branchen mit den geringsten Werten beim Digitalisierungsindex gehört, auch ein überdurchschnittliches Ergebnis, nämlich in der Kategorie Geschäftsmodelle. Besonders gut schneidet diese Branche beim Indikator digitale Absatzkanäle ab. So werden hier beispielsweise 18,9\% der Absätze über den Kanal Online-Marktplätze Dritter getätigt, im Vergleich zu 8,6\% im Durchschnitt der Branchen. Aber auch der Tourismus hat hinsichtlich der Digitalisierung noch deutlichen Nachholbedarf. So weisen die Kategorien Forschungsund Innovationsaktivitäten, Innovationslandschaft sowie Qualifizierung sehr niedrige Werte auf.

Die übrigen betrachteten Branchen weisen sehr unterschiedliche Stärken und Schwächen in der Digitalisierung auf. Der Handel sowie die Branchengruppe Verkehr und Logistik schneiden bei der Digitalisierung ihrer Prozesse zwar überdurchschnittlich ab. Der Handel fällt jedoch insbesondere bei seinen Produkten, im Bereich der Qualifizierung der Mitarbeitenden, der Innovationslandschaft und der Aktivität im Bereich von Forschung und Entwicklung im Vergleich ab. Für die drei letztgenannten Kategorien gilt dies auch für die Branchengruppe Verkehr und Logistik.
Fazit

Grundsätzlich gilt es bei der Ergebnisinterpretation solcher Branchenvergleiche, die Besonderheiten der jeweiligen Branche zu berücksichtigen. Die Voraussetzungen für hohe Werte im Index sind in den Branchen unterschiedlich. Neben den oben bereits erwähnten Einschränkungen in der Branchengruppe Grundstoffe, Chemie und Pharma in der Kategorie Produkte gilt dies etwa im Handel in ähnlicher Weise. Das bedeutet jedoch nicht, dass ein solcher Vergleich nicht sinnvoll ist. Dies gilt insbesondere für den ab dem Erhebungsjahr 2021 möglichen intertemporalen Vergleich. Dieser wird aufzeigen, inwieweit die verschiedenen Branchengruppen, gegeben ihre Unterschiedlichkeit, sich dynamisch weiterentwickeln und die Potenziale der Digitalisierung heben.

Angesichts der in Teilen dennoch erheblichen Abstände in den Resultaten der einzelnen Branchen ist es für die nächsten Jahre wichtig, die Digitalisierung der Wirtschaft am Standort Deutschland insgesamt voranzutreiben und gleichzeitig eine Verringerung der Abstände zwischen den Branchen anzustreben. Es sollte kein Fokus auf Fortschritte der Digitalisierung einzelner Branchen gelegt werden, da Digitalisierung über Netzwerke funktioniert: Damit ein Wertschöpfungsnetzwerk - ehemals eine Wertschöpfungskette - Fortschritte in der Digitalisierung machen kann, müssen die einzelnen Bestandteile dieses Netzwerks digitaler werden. Da in derartigen Netzwerken oft verschiedene Branchen involviert sind, muss gelten, die Potenziale in allen Branchen zu heben, um die deutsche Wirtschaft bei der Digitalisierung insgesamt voranzubringen.

\section{Literatur}

Azkan, C., V. Demary, M. Fritsch, H. Goecke, T. Korte, A. Krotova, K. Lichtblau, E. Schmitz (2019), Readiness Data Economy. Bereitschaft der deutschen Unternehmen zur Teilhabe an der Datenwirtschaft, Gutachten im Auftrag des Bundesministeriums für Wirtschaft und Energie, DEMAND-Projekt (demand-projekt.de) (12. Januar 2021).

Büchel, J., V. Demary, B. Engels, H. Goecke, C. Rusche (2020), Digitalisierung der Wirtschaft. Methodik des Digitalisierungsindex, Gutachten im Auftrag des Bundesministeriums für Wirtschaft und Energie, DE.DIGITAL - Methodik des Digitalisierungsindex (7. Januar 2021).

Europäische Kommission (2020), The Digital Economy and Society Index (DESI), https://ec.europa.eu/digital-single-market/en/news/digitaleconomy-and-society-index-desi-2020 (12. Januar 2021)

Title: Digitalization of Industries in Germany - An Empirical Survey

Abstract: The digitalisation index presented in this paper measures the degree of digitalisation of companies in Germany, differentiating between industries and classifying the degree of digitisation into categories. It shows that information and communication technology is by far the most digitised sector. Vehicle manufacturing, which is a strong research sector, is also highly digitised. Tourism and other manufacturing bring up the rear.

JEL Classification: C43, L00, 033, 052 